\title{
Reduction of boundary effects in multiple image deconvolution with an application to LBT LINC-NIRVANA
}

\author{
B. Anconelli ${ }^{1}$, M. Bertero ${ }^{1}$, P. Boccacci ${ }^{1}$, M. Carbillet $^{2}$, and H. Lanteri ${ }^{2}$ \\ 1 DISI, Università di Genova, Via Dodecaneso 35, 16146 Genova, Italy \\ ${ }^{2}$ Laboratoire Universitaire d'Astrophysique de Nice, UMR 6525, Parc Valrose, 06108 Nice Cedex 02, France
}

Received 18 July 2005 / Accepted 21 October 2005

\begin{abstract}
Our approach proposed in a previous paper for the reduction of boundary effects in the deconvolution of astronomical images by the RichardsonLucy method (RLM) is extended here to the problem of multiple image deconvolution and applied to the reconstruction of the images of LINC-NIRVANA, the German-Italian beam combiner for the Large Binocular Telescope (LBT). We investigate the multiple image RLM, its accelerated version ordered subsets expectation maximization (OSEM), and the regularized versions of these two methods. In addition we show how the approach can be extended to the iterative space reconstruction algorithm (ISRA), which is an iterative method converging to nonnegative least squares solutions. Numerical simulations indicate that the approach can provide excellent results with a considerable reduction of the boundary effects.
\end{abstract}

Key words. methods: data analysis - methods: numerical

\section{Introduction}

In a previous paper (Bertero \& Boccacci 2005) we proposed implementation of the Richardson-Lucy method (RLM) (Richardson 1972; Lucy 1974) that allowed for a reduction of the boundary effects; the same idea has been subsequently applied by Vio et al. (2005) to the Landweber method, in the framework of least squares solutions. Just after publication of our paper we discovered that a very similar approach was proposed years ago and implemented in the lucy task of STSDAS (White 1993; Stobie et al. 1994), even if a complete description of the implementation details was not provided in the refereed literature. The approach, derived from ideas developed by Politte \& Snyder (1991) in the case of medical imaging, was based on the use of a weight array compensating for the flat field and masking the bad pixels, as well as the outer parts of the image.

We recall that boundary effects appear if the astronomical object contained in the field of view (FOV) of the image is not surrounded by free sky and if FFT-based deconvolution algorithms are used. Indeed, FFT implicitly assumes a periodic continuation of the image, which is discontinuous at the boundary of the original domain. In the deconvolved image these discontinuities generate Gibbs oscillations (sometimes called ripples), which propagate inside the image domain and completely degrade the quality of the reconstruction.

Starting from the observation that, due to the extent of the point spread function (PSF), the detected image receives contributions from a domain that is a bit broader than the FOV of the telescope, the basic idea of our method consists in also attempting a reconstruction of the object outside the image domain. In this approach the number of unknowns (the pixel values of the object) is greater than the number of data (the pixel values of the image), so that it seems impossible to use FFT-based methods: the problem becomes rapidly intractable from the numerical point of view when the size of the image increases. However, an efficient implementation of this idea, in the case of RLM, was proposed in Bertero \& Boccacci (2005). If the image is $N \times N$, then our approach essentially leads to a FFT-based RLM applied to $2 N \times 2 N$ arrays, with an increase in the computational cost compared to standard RLM by a factor of about 16; indeed, the increase in the FFT computational cost is about 4 , and each RLM iteration requires the computation of 4 FFTs. It must also be pointed out that our approach does not require a symmetric PSF.

An alternative is provided by the methods based on the use of boundary conditions (BC), which extend the object outside the FOV in a somewhat artificial way. Even if an efficient implementation of these methods was originally proposed only in the case of symmetric PSFs (Chan et al. 1999; Serra Capizzano 2003), it has been recently noted (Donatelli \& Serra Capizzano 2005) that this requirement is not strictly necessary. Therefore a comparison between ours and the BC-based methods is possible, in principle, for any kind of PSF, and the choice between one or the other method is not determined by computational efficiency but, rather, by accuracy in the reconstruction of the object. 
The purpose of this paper is to extend our method to the deconvolution of multiple interferometric images of the same astronomical target. Our group has already developed methods and software for the solution of this problem (Bertero \& Boccacci 2000a,b; Correia et al. 2002; Carbillet et al. 2002) and produced the software package AIRY (Astronomical Image Reconstruction in interferometrY), for which version 3.0 is now available (see http://dirac.disi.unige.it and http://www.arcetri.astro.it/caos). This tool can be applied to Fizeau interferometers such as LINC-NIRVANA (Lbt INterferometric Camera and Near-InfraRed/Visible Adaptive iNterferometer for Astronomy), the German-Italian beam combiner for the Large Binocular Telescope (LBT). We recall that LBT will consist of two $8.4 \mathrm{~m}$ mirrors on a common mount, with a spacing of $14.4 \mathrm{~m}$ between their centers, so that a maximum baseline of $22.8 \mathrm{~m}$ will be available. The first light of the first primary mirror has been achieved on October 12 , 2005.

The paper is organized as follows. In Sect. 2 our approach is extended to multiple-image RLM and its accelerated version ordered subset expectation maximization (OSEM) (Bertero \& Boccacci 2000a), while in Sect. 3 it is extended to regularized versions of the same methods. In Sect. 4 we discuss its application to the least-squares (LS) method known as iterative space reconstruction algorithm (ISRA) and, finally, in Sect. 5 we present the results of a few numerical simulations in the case of LBT LINC-NIRVANA. Section 6 is devoted to the discussion of the perspectives of the proposed method.

\section{Multiple image RLM and OSEM}

Let $\boldsymbol{g}_{j},(j=1, \ldots, p)$ be $p$ different images of the same astronomical target. For instance, in the case of LBT LINCNIRVANA, they are obtained by means of different orientations of the baseline. Moreover, let us assume that the $\boldsymbol{g}_{j}$ are $N \times N$ arrays corresponding to the FOV of the detector, hence to a given angular region of the sky. These images receive contributions from a broader region, so that we must take a broader array for describing the astronomical object that contributes to the images. The size of this array depends on the size of the PSFs and therefore depends on the particular telescope we are considering. For this reason and also for using dimensions that are a power of 2 , we consider arrays $2 N \times 2 N$ and look for methods that leave the choice of the reconstruction domain to the particular PSFs we are using. We will denote the $2 N \times 2 N$ arrays by a bar, and the multi-index characterizing the elements of these arrays by $\boldsymbol{n}=\left\{n_{1}, n_{2}\right\}$.

The ingredients of the method are the following.

- $\overline{\boldsymbol{g}}_{j},(j=1, \ldots, p)$ are $2 N \times 2 N$ arrays, obtained by zero padding from the original images $\boldsymbol{g}_{j}$. Since the use of the FFT implies a periodic continuation of these arrays, the location of $\boldsymbol{g}_{j}$ within the corresponding $\overline{\boldsymbol{g}}_{j}$ is not important; for instance, they can be located in the central region or in the lower-left quadrant. Either way, the choice must be the same for all the $p$ images.
$-\bar{S}$ is the full domain of the values of the multi-index $\mathbf{n}$, while $S \subset \bar{S}$ is the subset of values corresponding to the detected images, hence to the pixels of the FOV.

- $\overline{\boldsymbol{b}}_{j},(j=1, \ldots, p)$ are $2 N \times 2 N$ uniform arrays, formed with the constant values of the backgrounds.

- $\overline{\boldsymbol{f}}$ is the $2 N \times 2 N$ array of the unknown values of the astronomical target. We denote by $\boldsymbol{f}$ the restriction of $\overline{\boldsymbol{f}}$ to $S$, namely to the FOV of the detected images.

- $\overline{\boldsymbol{K}}_{j}$ are $2 N \times 2 N$ PSFs, obtained by zero padding and shifting from the original PSFs (extracted, for instance, from the images of a star in the FOV).

We will denote the matrix associated to the PSF $\overline{\boldsymbol{K}}_{j}$ by $\bar{A}_{j}$, and by $\bar{A}_{j}^{T}$ the corresponding transposed matrix:

$$
\begin{aligned}
& \left(\bar{A}_{j} \overline{\boldsymbol{f}}\right)(\boldsymbol{n})=\sum_{\boldsymbol{n}^{\prime} \in \bar{S}} \overline{\boldsymbol{K}}_{j}\left(\boldsymbol{n}-\boldsymbol{n}^{\prime}\right) \overline{\boldsymbol{f}}\left(\boldsymbol{n}^{\prime}\right), \\
& \left(\bar{A}_{j}^{T} \overline{\boldsymbol{g}}\right)\left(\boldsymbol{n}^{\prime}\right)=\sum_{\boldsymbol{n} \in \bar{S}} \overline{\boldsymbol{K}}_{j}\left(\boldsymbol{n}-\boldsymbol{n}^{\prime}\right) \overline{\boldsymbol{g}}(\boldsymbol{n}) .
\end{aligned}
$$

Both can be computed by means of the FFT. As usual, we assume that the PSFs satisfy the normalization condition:

$\sum_{\boldsymbol{n}^{\prime} \in \bar{S}} \overline{\boldsymbol{K}}_{j}\left(\boldsymbol{n}^{\prime}\right)=1$,

which will be used in the following.

As shown by Shepp \& Vardi (1982), RLM is an iterative method for the maximization of the likelihood function if the images are contaminated by photon noise. In view of a unified approach, which also applies to the LS method discussed in Sect. 4, we use the equivalence between the maximization of the likelihood function and the minimization of the Csiszár I-divergence (Csiszár 1991). In the case of multiple image deconvolution, this is given by (Anconelli et al. 2005):

$$
\begin{aligned}
I(\boldsymbol{f} \mid \boldsymbol{g})= & \sum_{j=1}^{p} \sum_{\boldsymbol{n} \in S}\left\{\boldsymbol{g}_{j}(\boldsymbol{n}) \ln \frac{\boldsymbol{g}_{j}(\boldsymbol{n})}{\left(A_{j} \boldsymbol{f}\right)(\boldsymbol{n})+\boldsymbol{b}_{j}(\boldsymbol{n})}\right. \\
& \left.+\left[\left(A_{j} \boldsymbol{f}\right)(\boldsymbol{n})+\boldsymbol{b}_{j}(\boldsymbol{n})-\boldsymbol{g}_{j}(\boldsymbol{n})\right]\right\} .
\end{aligned}
$$

The multiple image RLM, as derived in Bertero \& Boccacci (2000b), is just an iterative method for the minimization of this functional. It is closely related to the method proposed by Lucy \& Hook (1992) for combining images with possibly very different PSFs. For completeness we recall that this model can also be used for approximately including the effect of the read-out noise (Snyder et al. 1993, 1994, 1995).

In view of dealing with boundary effects, since $\bar{f}$ is now the unknown object, we replace the previous functional with the following one:

$$
\begin{aligned}
I(\overline{\boldsymbol{f}} \mid \overline{\boldsymbol{g}})= & \sum_{j=1}^{p} \sum_{\boldsymbol{n} \in \bar{S}}\left\{\overline{\boldsymbol{g}}_{j}(\boldsymbol{n}) \ln \frac{\overline{\boldsymbol{g}}_{j}(\boldsymbol{n})}{\left(\bar{A}_{j} \overline{\boldsymbol{f}}\right)(\boldsymbol{n})+\overline{\boldsymbol{b}}_{j}(\boldsymbol{n})}\right. \\
& \left.+M_{S}(\boldsymbol{n})\left[\left(\bar{A}_{j} \overline{\boldsymbol{f}}\right)(\boldsymbol{n})+\overline{\boldsymbol{b}}_{j}(\boldsymbol{n})-\overline{\boldsymbol{g}}_{j}(\boldsymbol{n})\right]\right\},
\end{aligned}
$$

where the definitions given above are used and, as usual, we take $x \ln x=0$ if $x=0$. The array $\boldsymbol{M}_{S}(\boldsymbol{n})$ is the mask of the 
set $S$ (i.e. the array that is 1 on $S$ and 0 outside); this mask is not indicated in the denominator of the argument of the logarithm because the quotient is automatically zero outside $S$ thanks to the numerator. We point out that:

- the functional depends on $2 N \times 2 N$ unknowns;

- only the detected images and the restrictions to $S$ of the computed images $\bar{A}_{j} \bar{f}$ and of the backgrounds $\overline{\boldsymbol{b}}_{j}$ contribute to the functional.

It is obvious that the functional can be written without introducing the zero values of $\overline{\boldsymbol{g}}_{j}$ and the mask of the domain $S$; but, if we write the functional in the form of Eq. (4), then all quantities containing the imaging matrices $\bar{A}_{j}$ can be computed by means of the FFT.

Necessary and sufficient conditions for an array $\bar{f}$ to be a minimum of the convex functional $I(\overline{\boldsymbol{f}} \mid \overline{\boldsymbol{g}})$ in the cone of the non-negative arrays are provided by the well-known Kuhn-Tucker conditions (KTC):

$\overline{\boldsymbol{f}}\left(\boldsymbol{n}^{\prime}\right)\left[\nabla_{\bar{f}} I(\overline{\boldsymbol{f}} \mid \bar{g})\right]\left(\boldsymbol{n}^{\prime}\right)=0, \boldsymbol{n}^{\prime} \in \bar{S}$,

$\overline{\boldsymbol{f}}\left(\boldsymbol{n}^{\prime}\right) \geq 0,\left[\nabla_{\bar{f}} I(\overline{\boldsymbol{f}} \mid \overline{\boldsymbol{g}})\right]\left(\boldsymbol{n}^{\prime}\right) \geq 0$.

Then, by defining products and quotients of arrays as pixel by pixel products and quotients, the gradient of the functional of Eq. (4) can be written in the compact form:

$\nabla_{\overline{\boldsymbol{f}}} I(\overline{\boldsymbol{f}} \mid \overline{\boldsymbol{g}})=-\sum_{j=1}^{p} \bar{A}_{j}^{T} \frac{\overline{\boldsymbol{g}}_{j}}{\bar{A}_{j} \overline{\boldsymbol{f}}+\overline{\boldsymbol{b}}_{j}}+\bar{\alpha}$,

$\bar{\alpha}\left(\boldsymbol{n}^{\prime}\right)=\sum_{j=1}^{p} \bar{\alpha}_{j}\left(\boldsymbol{n}^{\prime}\right), \boldsymbol{n}^{\prime} \in \bar{S}$,

$\bar{\alpha}_{j}\left(\boldsymbol{n}^{\prime}\right)=\sum_{\mathbf{n} \in \bar{S}} \boldsymbol{M}_{S}(\boldsymbol{n}) \overline{\boldsymbol{K}}_{j}\left(\boldsymbol{n}-\boldsymbol{n}^{\prime}\right)=\left(\bar{A}_{j}^{T} \boldsymbol{M}_{S}\right)\left(\boldsymbol{n}^{\prime}\right)$.

If the PSFs satisfy the normalization condition of Eq. (2), then all the weight functions $\bar{\alpha}_{j}\left(\boldsymbol{n}^{\prime}\right)$ are approximately 1 in the central region of $S$, so that the weight function $\bar{\alpha}\left(\boldsymbol{n}^{\prime}\right)$ is approximately $p$ in the same domain. We point out that the arrays $\bar{\alpha}_{j}$ can be computed by means of the FFT.

From the previous equations it follows that the first KTC becomes:

$\bar{\alpha} \overline{\boldsymbol{f}}=\overline{\boldsymbol{f}}\left(\sum_{j=1}^{p} \bar{A}_{j}^{T} \frac{\overline{\boldsymbol{g}}_{j}}{\bar{A}_{j} \overline{\boldsymbol{f}}+\overline{\boldsymbol{b}}_{j}}\right)$.

Since the PSFs $\overline{\boldsymbol{K}}_{j}$ have been obtained by zero padding from the original PSFs (whose extent can be smaller than $S$ ), the weighting function $\bar{\alpha}$ can be zero in several pixels and, in the same pixels, the sum at the r.h.s. of this equation is also zero. It follows that, in these pixels, the values of the object are not determined by Eq. (10). We can decide to set these values to zero. More generally we can decide to set to zero the values of the object in the pixels where the weighting function $\bar{\alpha}$ is too small. Therefore we can search for solutions satisfying this constraint.

As in Bertero \& Boccacci (2005) we define a reconstruction domain $R \supset S$ as follows: consider a threshold $\sigma<1$, then $R$ is the set where $\bar{\alpha} / p>\sigma$ (we can take, for instance, $\sigma=10^{-2}$ or even smaller). It is obvious that $R$ depends on $\sigma$ but, for simplicity, we omit this dependence. The zeroing of $\bar{f}$ outside $R$ can be obtained by introducing the following window:

$$
\overline{\boldsymbol{w}}_{R}\left(\boldsymbol{n}^{\prime}\right)= \begin{cases}\frac{p}{\bar{\alpha}\left(\boldsymbol{n}^{\prime}\right)}, & \text { if } \frac{\bar{\alpha}\left(\boldsymbol{n}^{\prime}\right)}{p}>\sigma, \\ 0, & \text { otherwise, }\end{cases}
$$

which is approximately 1 in the central part of $S$, and by modifying Eq. (10) in the following way:

$\overline{\boldsymbol{f}}=\frac{1}{p} \overline{\boldsymbol{w}}_{R} \overline{\boldsymbol{f}}\left(\sum_{j=1}^{p} \bar{A}_{j}^{T} \frac{\overline{\boldsymbol{g}}_{j}}{\bar{A}_{j} \overline{\boldsymbol{f}}+\overline{\boldsymbol{b}}_{j}}\right)$.

If we look at this relation as a fixed point equation, then RLM can be formally obtained by applying the method of successive approximations. The result is as follows:

$\overline{\boldsymbol{f}}^{(k+1)}=\frac{1}{p} \overline{\boldsymbol{w}}_{R} \overline{\boldsymbol{f}}^{(k)}\left(\sum_{j=1}^{p} \bar{A}_{j}^{T} \frac{\overline{\boldsymbol{g}}_{j}}{\bar{A}_{j} \overline{\boldsymbol{f}}^{(k)}+\overline{\boldsymbol{b}}_{j}}\right)$,

and all the iterates are automatically zero outside $R$. Moreover, in order to extract one specific minimum of $I(\overline{\boldsymbol{f}} \mid \overline{\boldsymbol{g}})$, we take it as a rule to initialize the iterations with a constant array.

As already observed, if the background is not zero, then the denominator is never zero and the quotient is zero outside $S$ thanks to the numerator. However, if the background is zero, then the term $\bar{A}_{j} \bar{f}^{(k)}$ can be zero in pixels outside $S$, as an effect of the zero padding of the PSFs. In such a case, it is possible to avoid the undefined quotient $0 / 0$ by writing the algorithm as in Eq. (13), with the background replaced by the mask of the set $\bar{R}=\bar{S}-R$, i.e. the array that is 0 over $R$ and 1 outside.

Equation (13) extends the method proposed in Bertero \& Boccacci (2005) to the problem of multiple-image deconvolution. However, before introducing the OSEM algorithm for faster implementation of this method, we discuss one normalization problem.

From Eq. (13) it is easy to show by means of standard arguments that, if $\overline{\boldsymbol{b}}_{j}=0$ for any $j$, then all the iterates satisfy the condition:

$\frac{1}{p} \sum_{\boldsymbol{n}^{\prime} \in R} \bar{\alpha}\left(\boldsymbol{n}^{\prime}\right) \overline{\boldsymbol{f}}^{(k)}\left(\boldsymbol{n}^{\prime}\right)=\frac{1}{p} \sum_{j=1}^{p} \sum_{\boldsymbol{n} \in S} \boldsymbol{g}_{j}(\boldsymbol{n})$.

The interpretation of this relation is the following: $\bar{\alpha}\left(\boldsymbol{n}^{\prime}\right) / p$ gives the fraction of photons emitted at pixel $\boldsymbol{n}^{\prime}$, which contributes to the average flux of the detected images. When the backgrounds are not zero, the iterates do not satisfy this condition but they approximately satisfy a similar one that is obtained by replacing the 1.h.s. of Eq. (14) with the following quantity:

$c=\frac{1}{p} \sum_{j=1}^{p} \sum_{n \in S}\left\{\boldsymbol{g}_{j}(\boldsymbol{n})-\boldsymbol{b}_{j}(\boldsymbol{n})\right\}$.

Therefore it is convenient to minimize the functional of Eq. (4) with the additional constraint:

$\frac{1}{p} \sum_{\boldsymbol{n}^{\prime} \in R} \bar{\alpha}\left(\boldsymbol{n}^{\prime}\right) \overline{\boldsymbol{f}}\left(\boldsymbol{n}^{\prime}\right)=c$. 
As shown by Lanteri et al. (2002) in a more general context, this constraint can be introduced by re-normalizing the result of each RLM iteration. Such an approach can be very useful, especially in the case of OSEM, in order to reduce possible oscillations of the iterates. Indeed, OSEM is formulated in terms of cycles over the $p$ images, each element of a cycle being a single-image RLM iteration.

The version of the OSEM algorithm, implementing the method for the reduction of the boundary effects, written in the form given in Anconelli et al. (2005) is as follows.

\section{Preprocessing step}

- Compute the flux constant $c$ defined in Eq. (15).

- For $j=1, \ldots p$ compute the windows functions $\overline{\boldsymbol{w}}_{R, j}$ defined by:

$$
\overline{\boldsymbol{w}}_{R, j}\left(\boldsymbol{n}^{\prime}\right)= \begin{cases}\frac{1}{\bar{\alpha}_{j}\left(\boldsymbol{n}^{\prime}\right)}, & \text { if } \boldsymbol{n}^{\prime} \in R, \\ 0, & \text { otherwise. }\end{cases}
$$

\section{Reconstruction step}

- Initialize the algorithm with $f^{(0)}=$ const., satisfying the constraint of Eq. (16).

- Given $\overline{\boldsymbol{f}}^{(k)}$, set $j=(k+1) \bmod p$, and compute:

$$
\begin{aligned}
\tilde{\boldsymbol{f}}^{(k+1)} & =\overline{\boldsymbol{w}}_{R, j} \overline{\boldsymbol{f}}^{(k)}\left(\bar{A}_{j}^{T} \frac{\overline{\boldsymbol{g}}_{j}}{\bar{A}_{j} \overline{\boldsymbol{f}}^{(k)}+\overline{\boldsymbol{b}}_{j}}\right), \\
\tilde{\boldsymbol{c}}^{(k+1)} & =\frac{1}{p} \sum_{\boldsymbol{n}^{\prime} \in R} \bar{\alpha}\left(\boldsymbol{n}^{\prime}\right) \tilde{\boldsymbol{f}}^{(k+1)}\left(\boldsymbol{n}^{\prime}\right) ;
\end{aligned}
$$

- Set:

$$
\overline{\boldsymbol{f}}^{(k+1)}=\frac{c}{\tilde{\boldsymbol{c}}^{(k+1)}} \tilde{\boldsymbol{f}}^{(k+1)} \text {. }
$$

All the algorithms derived in this section will be available in the software package AIRY, version 3.1.

\section{Regularized RLM and OSEM}

From the analysis in the previous section, it is clear that the object is reconstructed only inside the domain $R$; therefore, the method provides the minimization of the functional that is obtained from that of Eq. (4) by neglecting the contribution of the components of $\overline{\boldsymbol{f}}$ corresponding to pixels outside $R$. If we do not omit the dependence of the functional on these components for computational reasons, then it must be written in the following way:

$$
\begin{aligned}
I(\overline{\boldsymbol{f}} \mid \overline{\boldsymbol{g}})= & \sum_{j=1}^{p} \sum_{\boldsymbol{n} \in \bar{S}}\left\{\overline{\boldsymbol{g}}_{j}(\boldsymbol{n}) \ln \frac{\overline{\boldsymbol{g}}_{j}(\boldsymbol{n})}{\left(\bar{A}_{j} M_{R} \overline{\boldsymbol{f}}\right)(\boldsymbol{n})+\overline{\boldsymbol{b}}_{j}(\boldsymbol{n})}\right. \\
& \left.+M_{S}(\boldsymbol{n})\left[\left(\bar{A}_{j} M_{R} \overline{\boldsymbol{f}}\right)(\boldsymbol{n})+\overline{\boldsymbol{b}}_{j}(\boldsymbol{n})-\overline{\boldsymbol{g}}_{j}(\boldsymbol{n})\right]\right\},
\end{aligned}
$$

where $M_{R}$ is the mask of the reconstruction domain, namely the array that is 1 inside $R$ and 0 outside. In the RLM and OSEM algorithms, as described in the previous section, we can omit $M_{R}$ in the computation of $A_{j} M_{R} \overline{\boldsymbol{f}}^{(k)}$, because the multiplicative structure of the algorithms guarantees that $\overline{\boldsymbol{f}}^{(k)}$ is zero outside $R$.
The previous expression of $I(\overline{\boldsymbol{f}} \mid \overline{\boldsymbol{g}})$ is useful in view of the development of regularized versions of RLM and OSEM. In such a case the problem becomes the minimization of the following functional:

$J_{\mu}(\overline{\boldsymbol{f}} \mid \overline{\boldsymbol{g}})=I(\overline{\boldsymbol{f}} \mid \overline{\boldsymbol{g}})+\mu \Omega\left(M_{R} \overline{\boldsymbol{f}}\right)$,

where the first term is that defined in Eq. (20) and the second one is the regularization functional; $\mu$ is the regularization parameter. Since this functional does not depend on the components of $\bar{f}$ corresponding to pixels outside $R$, the corresponding components of the gradient are zero. As a consequence, we can write the gradient of the first term in the following form, derived from Eq. (7):

$$
\begin{aligned}
\nabla_{\overline{\boldsymbol{f}}} I(\overline{\boldsymbol{f}} \mid \overline{\boldsymbol{g}})= & -M_{R}\left(\sum_{j=1}^{p} \bar{A}_{j}^{T} \frac{\overline{\boldsymbol{g}}_{j}}{\bar{A}_{j} M_{R} \overline{\boldsymbol{f}}+\overline{\boldsymbol{b}}_{j}}\right) \\
& +M_{R} \bar{\alpha} .
\end{aligned}
$$

We note that this is given by the difference of two non-negative arrays. Then, according to the split gradient method (SGM), proposed by Lanteri et al. $(2001,2002)$, we write the gradient of the regularization functional in a similar way:

$$
\nabla_{\bar{f}} \Omega\left(M_{R} \bar{f}\right)=-M_{R} U_{\Omega}\left(M_{R} \bar{f}\right)+M_{R} V_{\Omega}\left(M_{R} \bar{f}\right),
$$

where $U_{\Omega}\left(M_{R} \bar{f}\right)$ and $V_{\Omega}\left(M_{R} \bar{f}\right)$ are two non-negative arrays. It is obvious that such a decomposition always exists even if it is not unique. Moreover, as shown in the above mentioned papers, realizations of the arrays $U_{\Omega}, V_{\Omega}$ can be computed easily for all the regularization functionals used in practice. For instance, in the case of the well-known Tikhonov functional

$\Omega\left(M_{R} \bar{f}\right)=\frac{1}{2}\left\|M_{R} \bar{f}\right\|^{2}$,

we have

$U_{\Omega}\left(M_{R} \bar{f}\right)=0, V_{\Omega}\left(M_{R} \bar{f}\right)=M_{R} \bar{f}$,

since we are considering the restriction of the functional to the cone of the non-negative arrays.

In terms of the previous expressions, the first KTC becomes:

$$
\begin{array}{r}
M_{R} \overline{\boldsymbol{f}}\left\{\sum_{j=1}^{p} \bar{A}_{j}^{T} \frac{\overline{\boldsymbol{g}}_{j}}{\bar{A}_{j} M_{R} \overline{\boldsymbol{f}}+\overline{\boldsymbol{b}}_{j}}+\mu U_{\Omega}\left(M_{R} \overline{\boldsymbol{f}}\right)\right\}= \\
M_{R} \overline{\boldsymbol{f}}\left\{\bar{\alpha}+\mu V_{\Omega}\left(M_{R} \overline{\boldsymbol{f}}\right)\right\} .
\end{array}
$$

Then, if we note that inside $R$ we can divide both sides of this equation by $\bar{\alpha}$, we obtain the following expression in terms of the window function of Eq. (11)

$$
\begin{array}{r}
\frac{1}{p} \overline{\boldsymbol{w}}_{R} \overline{\boldsymbol{f}}\left\{\sum_{j=1}^{p} \bar{A}_{j}^{T} \frac{\overline{\boldsymbol{g}}_{j}}{\bar{A}_{j} \overline{\boldsymbol{f}}+\overline{\boldsymbol{b}}_{j}}+\mu U_{\Omega}(\overline{\boldsymbol{f}})\right\}= \\
\overline{\boldsymbol{f}}\left\{I+\frac{\mu}{p} \overline{\boldsymbol{w}}_{R} V_{\Omega}(\overline{\boldsymbol{f}})\right\},
\end{array}
$$

where we do not indicate the mask $M_{R}$ because the factor $\overline{\boldsymbol{w}}_{R}$ automatically implies that each $\overline{\boldsymbol{f}}$ that satisfies this condition is zero outside $R$. 
If we divide both sides of Eq. (27) by the positive array $I+\frac{\mu}{p} \overline{\boldsymbol{w}}_{R} V_{\Omega}(\bar{f})$, we obtain a fixed-point equation that is a generalization of that of Eq. (12). Then, by applying the method of successive approximations, we get the regularized version of the RLM algorithm for multiple-image deconvolution with reduction of the boundary effects:

$$
\begin{aligned}
\overline{\boldsymbol{f}}^{(k+1)}= & \frac{1}{p} \overline{\boldsymbol{w}}_{R} \frac{\overline{\boldsymbol{f}}^{(k)}}{I+\frac{\mu}{p} \overline{\boldsymbol{w}}_{R} V_{\Omega}\left(\overline{\boldsymbol{f}}^{(k)}\right)} \\
& \times\left\{\sum_{j=1}^{p} \bar{A}_{j}^{T} \frac{\overline{\boldsymbol{g}}_{j}}{\bar{A}_{j} \overline{\boldsymbol{f}}^{(k)}+\overline{\boldsymbol{b}}_{j}}+\mu U_{\Omega}\left(\overline{\boldsymbol{f}}^{(k)}\right)\right\} .
\end{aligned}
$$

Again, the OSEM method is obtained by replacing one multiple-image RLM iteration by a cycle of $p$ single-image iterations:

$$
\begin{aligned}
\overline{\boldsymbol{f}}^{(k+1)}= & \overline{\boldsymbol{w}}_{R, j} \frac{\overline{\boldsymbol{f}}^{(k)}}{I+\mu \overline{\boldsymbol{w}}_{R, j} V_{\Omega}\left(\overline{\boldsymbol{f}}^{(k)}\right)} \\
& \times\left\{\bar{A}_{j}^{T} \frac{\overline{\boldsymbol{g}}_{j}}{\bar{A}_{j} \overline{\boldsymbol{f}}^{(k)}+\overline{\boldsymbol{b}}_{j}}+\frac{\mu}{p} U_{\Omega}\left(\overline{\boldsymbol{f}}^{(k)}\right)\right\},
\end{aligned}
$$

where $j=k+1 \bmod p$. We do not write the complete algorithm because it can be easily deduced from the one given at the end of Sect. 2.

\section{Multiple-image ISRA}

As already remarked in Bertero \& Boccacci (2005), the method based on the immersion, by zero padding, of the detected image into broader arrays can also be applied to least-squares (LS) iterative methods. However, in such a case, the increase in the computational cost is due not only to the increased size of the images to be processed but also to the increase in the number of FFTs per iteration. We discuss this point below.

The application of our approach to the Landweber method has been already discussed by Vio et al. (2005). Here we consider the application to ISRA. For generality, we give the equations in the multiple-image case but, of course, the reduction to the single-image case is obvious.

The starting point is the minimization of the well-known discrepancy functional

$$
I(\overline{\boldsymbol{f}} \mid \overline{\boldsymbol{g}})=\sum_{j=1}^{p}\left\|M_{S}\left(\bar{A}_{j} \overline{\boldsymbol{f}}+\overline{\boldsymbol{b}}_{j}\right)-\overline{\boldsymbol{g}}_{j}\right\|^{2}
$$

where the notations introduced in the previous sections are used and II.\| denotes the usual Euclidean norm of $2 N \times 2 N$ arrays. We notice that only the terms $\bar{A}_{j} \overline{\boldsymbol{f}}+\overline{\boldsymbol{b}}_{j}$ are multiplied by the mask $M_{S}$, because $M_{S} \overline{\boldsymbol{g}}_{j}=\overline{\boldsymbol{g}}_{j}$ for any $j$.

Since we are again considering minimization onto the non-negative cone, the KTCs provide the necessary and sufficient conditions to be satisfied by the minimum points. By taking into account that the gradient of the functional is given by:

$$
\nabla_{\bar{f}} I(\overline{\boldsymbol{f}} \mid \overline{\boldsymbol{g}})=2 \sum_{j=1}^{p}\left\{\bar{A}_{j}^{T} M_{S} \bar{A}_{j} \overline{\boldsymbol{f}}-\bar{A}_{j}^{T}\left(\overline{\boldsymbol{g}}_{j}-M_{S} \overline{\boldsymbol{b}}_{j}\right)\right\},
$$

the first KTC provides the following equation for the nonnegative minima:

$$
\overline{\boldsymbol{f}} \sum_{j=1}^{p} \bar{A}_{j}^{T}\left(M_{S} \bar{A}_{j} \overline{\boldsymbol{f}}+M_{S} \overline{\boldsymbol{b}}_{j}\right)=\overline{\boldsymbol{f}} \sum_{j=1}^{p} \bar{A}_{j}^{T} \overline{\boldsymbol{g}}_{j}
$$

In order to write this condition as a fixed-point equation, we have to face a difficulty similar to the one discussed in the case of Eq. (10). Indeed, since the PSFs $\overline{\boldsymbol{K}}_{j}$ have been obtained by zero padding, the sums appearing on both sides of Eq. (32) can be zero in pixels outside $S$ and, in these pixels, the object $\bar{f}$ is not determined. In other words, we again have the problem of defining a reconstruction domain $R \supset S$.

This domain can be obtained by a suitable thresholding, outside $S$, of the sum on the r.h.s. of Eq. (32). If we denote the average flux per pixel by $\bar{c}$

$\bar{c}=\frac{1}{N^{2}} \sum_{j=1}^{p} \sum_{n \in S} \boldsymbol{g}_{j}(\boldsymbol{n})$,

then given $\sigma<1$, we define $R$ as the union of $S$ and of the pixels outside $S$ where the following condition is satisfied:

$\sum_{j=1}^{p} \bar{A}_{j}^{T} \overline{\boldsymbol{g}}_{j}(\boldsymbol{n})>\sigma \bar{c}$.

With this definition of the reconstruction domain $R$, we can derive the following fixed-point equation from Eq. (32):

$\overline{\boldsymbol{f}}=\overline{\boldsymbol{f}} \frac{M_{R} \sum_{j=1}^{p} \bar{A}_{j}^{T} \overline{\boldsymbol{g}}_{j}}{\sum_{j=1}^{p} \bar{A}_{j}^{T}\left(M_{S} \bar{A}_{j} \overline{\boldsymbol{f}}^{\prime}+M_{S} \overline{\boldsymbol{b}}_{j}\right)}$,

where $M_{R}$ again denotes the mask of $R$. Then ISRA can be obtained by applying the successive approximation method to this equation. The result is the following:

$\overline{\boldsymbol{f}}^{(k+1)}=\overline{\boldsymbol{f}}^{(k)} \frac{M_{R} \sum_{j=1}^{p} \bar{A}_{j}^{T} \overline{\boldsymbol{g}}_{j}}{\sum_{j=1}^{p} \bar{A}_{j}^{T}\left(M_{S} \bar{A}_{j} \overline{\boldsymbol{f}}^{(k)}+M_{S} \overline{\boldsymbol{b}}_{j}\right)}$.

Since the denominator is computed in all the pixels of $\bar{S}$ and it can be zero in pixels outside $S$, we have a similar problem to the one already discussed in the case of RLM with zero background. Therefore, for algorithmic reasons, and in order to avoid undetermined forms $0 / 0$ outside $R$, we can add the mask of the domain $\bar{R}=\bar{S}-R$ to the denominator.

Also in the case of ISRA, it is convenient to normalize the result of each iteration; moreover, the OS-version is recommended in order to reduce the computational burden. The complete version of OS-ISRA is as follows:

\section{Preprocessing step}

- Compute the flux constant $c$ defined in Eq. (15).

- Compute the mask $M_{R}$ of the reconstruction domain by means of Eq. (34).

\section{Reconstruction step}

- Initialize the algorithm with $f^{(0)}=$ const., satisfying the constraint of Eq. (16). 

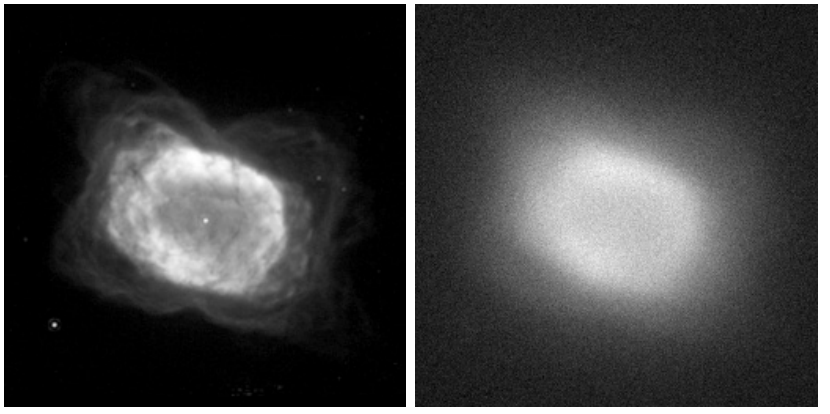

Fig. 1. The object used in our simulations (left) and one of the images obtained by convolving the object with one of the PSFs used in our numerical experiments (relative parallactic angle $=0^{\circ}, S R=26 \%$ ), and perturbing the result with Poisson and Gaussian noise (right).

- Given $\overline{\boldsymbol{f}}^{(k)}$, set $j=(k+1) \bmod p$, and compute:

$$
\begin{aligned}
\tilde{\boldsymbol{f}}^{(k+1)} & =\overline{\boldsymbol{f}}^{(k)} \frac{M_{R} \bar{A}_{j}^{T} \overline{\boldsymbol{g}}_{j}}{\bar{A}_{j}^{T}\left(M_{S} \bar{A}_{j} \overline{\boldsymbol{f}}^{(k)}+M_{S} \overline{\boldsymbol{b}}_{j}\right)+M_{\bar{R}}}, \\
\tilde{\boldsymbol{c}}^{(k+1)} & =\frac{1}{p} \sum_{\boldsymbol{n}^{\prime} \in R} \bar{\alpha}\left(\boldsymbol{n}^{\prime}\right) \tilde{\boldsymbol{f}}^{(k+1)}\left(\boldsymbol{n}^{\prime}\right) ;
\end{aligned}
$$

- Set:

$$
\overline{\boldsymbol{f}}^{(k+1)}=\frac{c}{\tilde{\boldsymbol{c}}^{(k+1)}} \tilde{\boldsymbol{f}}^{(k+1)} .
$$

The reduction of the boundary effects is due to the insertion of the mask $M_{S}$ in the algorithm. However, this mask increases the computational burden of ISRA. Indeed, one iteration of the standard version of ISRA requires the computation of 2 FFTs, hence, is cheaper than one iteration of RLM, that requires 4 FFTs. On the other hand, the computation of $\bar{A}_{j}^{T} M_{S} \bar{A}_{j}$ requires 4 FFTs; therefore, the computational cost of 1 OS-ISRA iteration with boundary effects reduction is just the same as one OSEM iteration.

\section{A numerical example}

In the previous sections we described several generalizations of the method proposed in Bertero \& Boccacci (2005) for the reduction of boundary effects in image deconvolution. In this section we discuss an application of the modified OSEM algorithm to simulated images of LINC-NIRVANA.

The object considered is the $256 \times 256$ HST image of the nebula NGC 7027, shown in the left panel of Fig. 1. The images were generated according to the model described, for instance, in Anconelli et al. (2005): the object was convolved with the simulated PSFs, sky backgrounds were added, and the results were perturbed with Poisson and Gaussian noise.

We first describe the three sets of PSFs used in our numerical experiments. All consist of three PSFs corresponding to three different orientations of the baseline with respect to the observed object (relative parallactic angle): $0^{\circ}, 60^{\circ}$, and $120^{\circ}$. The first set consists of ideal PSFs (not displayed), the second of AO-corrected PSFs with $S R=70 \%$, and the third of AO corrected PSFs with $S R=26 \%$. These PSFs were obtained by means of the software package CAOS (Code for Adaptive Optics Systems; Carbillet et al. 2005), according to the model
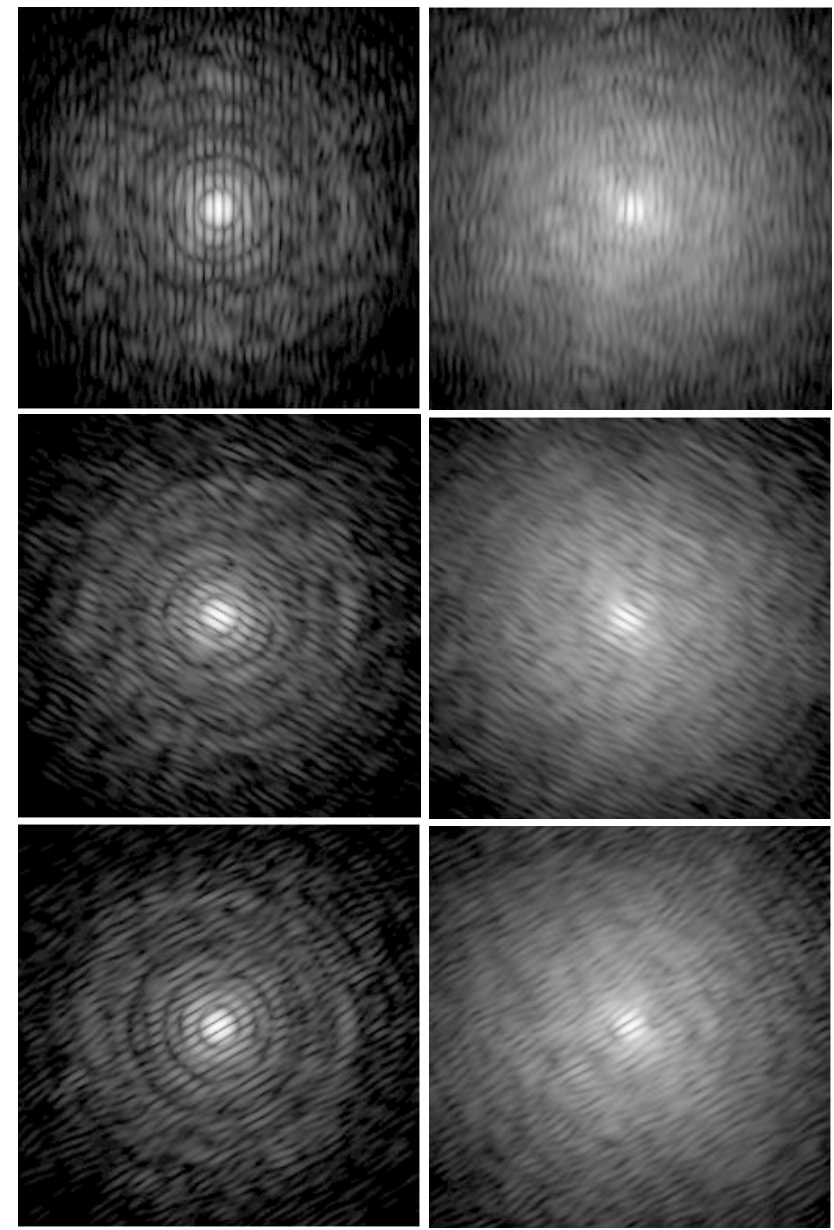

Fig. 2. The AO-corrected PSFs used in our simulations (logarithmic scale): in the left column the PSFs with Strehl ratio of about $70 \%$ and in the right column those with Strehl ration of about $26 \%$, for baseline orientations of $0^{\circ}, 60^{\circ}$, and $120^{\circ}$ (top to bottom).

already described in Carbillet et al. (2002) and Anconelli et al. (2005). The two sets of AO-corrected PSFs are displayed in Fig. 2: those with $S R=70 \%$ to the left and the others to the right.

Next, simulated $256 \times 256$ LINC-NIRVANA images were obtained by convolving the object, whose integrated magnitude in $K$-band was fixed to 15 , with the PSFs of the three sets described above. For the pixel size we assumed the value of the LINC-NIRVANA detector, namely 5.12 mas. Moreover, the following parameters were used in image generation: an integration time of $20 \mathrm{~min}$, a telescope surface of $104 \mathrm{~m}^{2}$, a sky background of $12.5 \mathrm{mag} / \operatorname{arcsec}^{2}$ ( $K$ band), a read out noise (RON) of $10 \mathrm{e}^{-} \mathrm{rms}$, and an efficiency of $25 \%$.

In a first set of experiments, we deconvolved the images by means of the same sets of PSFs used for their generation (inverse crime). Moreover each experiment, one for each set of PSFs, consisted of two parts:

- reconstruction of the complete $256 \times 256$ object using the standard version of OSEM from the software package AIRY; the result is a reference image that can be used for estimating the reduction of the boundary effects 

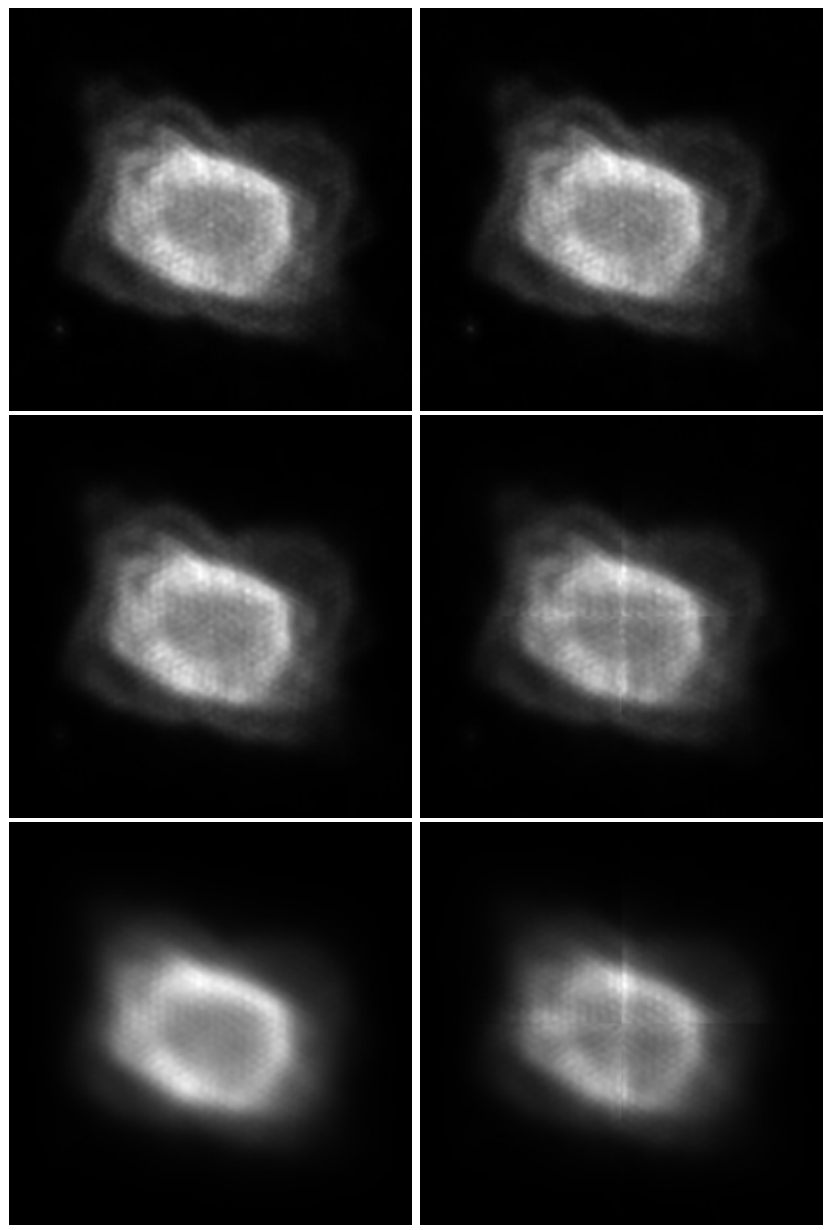

Fig. 3. Example of application of the method (inverse crime). In the left column we display the reconstructions obtained by deconvolving the complete images by means of standard OSEM and in the right column those obtained as a mosaic of the reconstructions of the four quadrants, as provided by the version of OSEM with reduction of the boundary effects. From top to bottom: ideal PSFs, AO-corrected PSFs with $S R=70 \%$ and with $S R=26 \%$.

provided by our method when it is used for reconstructing only a piece of this image;

- splitting of each image into four non-overlapping quadrants $128 \times 128$, reconstruction of each of them by means of the modified OSEM of Sect. 2, and finally, recombination of the four reconstructions (mosaic) to provide a reconstruction of the complete object; in this way it is possible to visualize the relevance of the boundary effects.

The results of these experiments are shown in Fig. 3.

In the upper panels we display the results obtained in the case of ideal PSFs. In the left panel we give the reconstruction obtained by deconvolving the full images; the minimum reconstruction error is about $0.5 \%$, and it is reached after 116 iterations (semi-convergence property of RLM or OSEM; see, for instance, Bertero \& Boccacci 1998). The reconstruction error was defined as the relative rms difference between the result of iteration $k, \boldsymbol{f}^{(k)}$, and the original object $\boldsymbol{f}$ :

$\rho^{(k)}=\frac{\left\|\boldsymbol{f}^{(k)}-\boldsymbol{f}\right\|}{\|\boldsymbol{f}\|}$.
Moreover, in the right panel we give the reconstruction obtained as a mosaic of the four reconstructions provided by the algorithm of Sect. 2, when applied to the four $128 \times 128$ quadrants of the detected images. For each quadrant we used 115 iterations and the reconstruction error, computed on the full $256 \times 256$ image, is about $0.6 \%$. This numerical result agrees with the fact that no visible difference exists between the left and right panels.

The situation is a bit different in the case of AO-corrected PSFs. In the middle panels we give the two reconstructions (global and mosaic) obtained in the case of PSFs with $S R=$ $70 \%$ and in the lower panels those obtained in the case of the PSFs with $S R=26 \%$. For each set of PSFs, the difference between the two different reconstructions is visible and increases with decreasing $S R$; this means that the reduction of boundary effects, provided by the method, is more significant in the case of ideal PSFs than in the case of AO-corrected PSFs. The reason may be that the ideal PSFs are symmetric, while the AO-corrected ones are not, and the symmetry breaking increases with decreasing $S R$.

More quantitative results are the following. In the case of $S R=70 \%$, the best global reconstruction was obtained after 120 iterations with a reconstruction error of about $0.7 \%$, while in the case $S R=26 \%$ the best reconstruction was obtained after 51 iterations with an error of about $3.5 \%$. Moreover, in the first case, the best mosaic is obtained after 79 iterations with an error of about $1.9 \%$, while, in the second case, the best mosaic was obtained after 30 iterations with an error of about $5.5 \%$.

In the last experiment we checked the robustness of the method with respect to errors on the PSFs. To this purpose we generated the images by convolving the object with AO-corrected PSFs $(S R=70 \%)$, and these images were subsequently deconvolved by using ideal PSFs. This experiment mimics a situation where the PSFs, corresponding to a high $S R$, are not known so one uses ideal PSFs for reconstructing the object. The result is shown in Fig. 4: in the left panel the global reconstruction is displayed, while the right panel shows the one obtained as a mosaic of the reconstructions of the four panels. In the first case the reconstruction error was $2.1 \%$ and was reached after 116 iterations; in the second case the reconstruction error was $2.2 \%$ and was reached after 110 iterations.

\section{Concluding remarks and perspectives}

In this paper we extend the method proposed in Bertero \& Boccacci (2005) for the reduction of boundary effects in image reconstruction to multiple-image deconvolution. The method is based on the idea of also reconstructing the object outside the domain of the detected images, while its implementation is based on the immersion by zero padding of the detected images $N \times N$ into broader ones, typically $2 N \times 2 N$. A few numerical experiments indicate that the method also works well in the case of multiple images of the same object and that it is robust with respect to errors in the PSFs used for the deconvolution.

The numerical experiments were performed with $128 \times$ 128 images. However, it must be observed that the detector of LINC-NIRVANA will produce $2048 \times 2048$ images and, in such a situation, it may not be convenient to double the size 

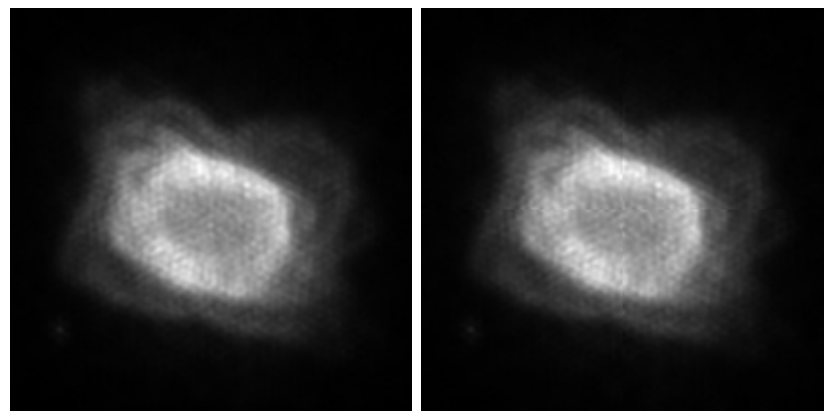

Fig. 4. Example of the application of the method without inverse crime. Convolution was performed with the AO-corrected PSFs $(S R=$ 70\%) and deconvolution with ideal PSFs. Left panel: reconstruction obtained by deconvolving the complete images by means of standard OSEM. Right panel: mosaic of the reconstructions of the four quadrants, as provided by the version of OSEM with reduction of the boundary effects.

of the images by zero padding as required in the version of the method presented in this paper. Moreover the domain of the PSFs will be much smaller than $2048 \times 2048$. For such big images the immersion should be in an array that is not much greater than the original one with a size that cannot be a power of 2. For instance, if the image is an $N \times N$ array and if the PSF is a $2 M \times 2 M$ array, then the immersion should be in an array $(N+M) \times(N+M)$. However, as is known, if the size of the array has a small prime factor, then the library FFTW (Fastest Fourier Transform in the West) could provide an efficient FFT. In the case of LINC-NIRVANA images, the closest array satisfying these conditions has a size $2187=\left(3^{7}\right)$, which is compatible with a $278 \times 278$ array for the PSFs.

An alternative could be provided by a deconvolution based on a domain decomposition of the images (Fish et al. 1996; Aubailly et al. 2004). This approach could also be required if the space variance of the PSFs is relevant. In general overlapping sub-domains are used, and only the reconstructions obtained in the central parts are kept, in order to reduce the boundary artifacts provided by the standard deconvolution methods. As shown by our numerical experiments, our method could be efficiently applied to each sub-domain, and since the boundary artifacts are considerably reduced by the method itself, slightly overlapping sub-domains could be used. Therefore we think that our approach can provide efficient deconvolution of large images. It is also obvious that a deconvolution based on a domain decomposition can be implemented easily on a multiprocessor system.
Acknowledgements. We thank Roberto Vio for providing us information about the previous work of Rick White and coworkers on the problem of boundary effects in image deconvolution. We also thank Rick White for reviewing and commenting this paper.

\section{References}

Aubailly, M., Roggemann, M. C., \& Schulz, T. 2004, Proc. 2004 AMOS Technical Conf., 131

Anconelli, B., Bertero, M., Boccacci, P., Carbillet, M., \& Lanteri, H. 2005, A\&A, 430, 731

Bertero, M., \& Boccacci, P. 1998, Introduction to Inverse Problems in Imaging (Bristol: IOP Publishing)

Bertero, M., \& Boccacci, P. 2000a, A\&AS, 144, 181

Bertero, M., \& Boccacci, P. 2000b, A\&AS, 147, 323

Bertero, M., \& Boccacci, P. 2005, A\&A, 437, 369

Carbillet, M., Correia, S., Boccacci, P., \& Bertero, M. 2002, A\&A, 387,744

Carbillet, M., Vérinaud, C., Femenía, B., et al. 2005, MNRAS, 356, 1263

Chan, R., Ng, M., \& Tang, W. C. 1999, SIAM J. Sci. Comput., 21, 851

Correia, S., Carbillet, M., Boccacci, P., Bertero, M., \& Fini, L. 2002, A\&A, 387, 733

Csiszár, I. 1991, Ann. Stat., 19, 2032

Donatelli, M., \& Serra Capizzano, S. 2005, Inverse Problems, 21, 169

Fish, D. A., Grochmalicki, J., \& Pike, E. R. 1996, JOSA, 13 A, 464

Lanteri, H., Roche, M., Cuevas, O., \& Aime, C. 2001, Signal Processing, 81, 945

Lanteri, H., Roche, M., \& Aime, C. 2002, Inverse Problems, 18, 1397

Lucy, L. B. 1974, AJ, 79, 745

Lucy, L. B., \& Hook, R. N. 1992, ASP Conf. Ser., 25, 277

Politte, D. G., \& Snyder, D. L. 1991, IEEE Trans. Med. Im., 10, 82

Richardson, W. H. 1972, JOSA, 62, 55

Snyder, D. L., Hammoud, A. M., \& White, R. L. 1993, JOSA A, 10, 1014

Snyder, D. L., Helstrom, C. W., Lanterman, A. D., Faisal, M., \& White, R. L. 1994, in The Restoration of HST Images and Spectra-II (Baltimore:STScI), 139

(http://www.stsci.edu/stsci/meetings/irw/

proceedings/snyderd.html)

Snyder, D. L., Helstrom, C. W., Lanterman, A. D., Faisal, M., \& White, R. L. 1995, JOSA A, 12, 272

Serra Capizzano, S. 2003, SIAM J. Sci. Comput., 25, 1307

Shepp, L. A., \& Vardi, Y. 1982, IEEE Trans. Med. Im., 1, 113

Stobie, E. B., Hanish, R. J., \& White, R. L. 1994, ASP Conf. Ser., 61, 296

Vio, R., Bardsley, J., Donatelli, M., \& Wamsteker, W. 2005, A\&A, 439, 1229

White, R. L. 1993, Newsletter STScI's Image Restoration Project, 1, 11 